\title{
ON THE SOLVABILITY OF THE MODIFIED CAUCHY PROBLEM FOR LINEAR SYSTEMS OF IMPULSIVE DIFFERENTIAL EQUATIONS WITH SINGULARITIES
}

\author{
MALKHAZ ASHORDIA AND NATO KHARSHILADZE
}

Received 24 February, 2020

\begin{abstract}
The modified Cauchy problem is considered for systems of linear impulsive differential equations with singularities. The singularity is considered in the sense that the matrix-and vector-functions corresponding to the impulsive system are generally not integrable at the initial point. The sufficient conditions are established for the unique solvability of the problem
\end{abstract}

2010 Mathematics Subject Classification: 34A12; 34A30; 34A37; 34K26

Keywords: impulsive differential equations, linear systems, singularities, the modified Cauchy problem, unique solvability

\section{STATEMENT OF THE PROBLEM AND BASIC NOTATION}

Let $I \subset \mathbb{R}$ be a non-degenerate interval in the point, $t_{0} \in I$ and

$$
I_{t_{0}}=I \backslash\left\{t_{0}\right\} .
$$

Consider the linear system of impulsive differential equations

$$
\begin{gathered}
\frac{d x}{d t}=P(t) x+q(t) \text { for a.a. } t \in I_{t_{0}} \backslash T, \\
x\left(\tau_{l}+\right)-x\left(\tau_{l}-\right)=G(l) x\left(\tau_{l}\right)+g(l) \quad(l=1,2, \ldots),
\end{gathered}
$$

where $T=\left\{\tau_{1}, \tau_{2}, \ldots\right\}, \tau_{l} \in I_{t_{0}}(l=1,2, \ldots)$ are points of impulses actions such that $\lim _{l \rightarrow+\infty} \tau_{l}=t_{0}$

$$
P=\left(p_{i k}\right)_{i, k=1}^{n} \in L_{l o c}\left(I_{t_{0}} ; \mathbb{R}^{n \times n}\right) \text { and } q=\left(q_{k}\right)_{k=1}^{n} \in L_{l o c}\left(I_{t_{0}} ; \mathbb{R}^{n}\right)
$$

are matrix- and vector-functions, respectively, and

$$
G=\left(g_{i k}\right)_{i, k=1}^{n} \in E\left(\mathbb{N} ; \mathbb{R}^{n \times n}\right) \text { and } g=\left(g_{k}\right)_{k=1}^{n} \in E\left(\mathbb{N} ; \mathbb{R}^{n}\right)
$$

are matrix- and vector-functions of the discrete argument.

Let $H=\operatorname{diag}\left(h_{1}, \ldots, h_{n}\right): I_{t_{0}} \rightarrow \mathbb{R}^{n \times n}$ be a diagonal matrix-function with continuous diagonal elements

$$
\left.h_{k}: I_{t_{0}} \rightarrow\right] 0,+\infty[(k=1, \ldots, n) .
$$


We consider the problem of finding of a solution $x: I_{t_{o}} \rightarrow R^{n}$ of system (1.1), (1.2), satisfying the condition

$$
\lim _{t \rightarrow t_{0}}\left(H^{-1}(t) x(t)\right)=0 .
$$

As we know firstly the initial problem for systems of ordinary differential equations with singularities have been fundamentally investigated by V.A. Chechik in [7], where the sufficient conditions for the existence and uniqueness of a solution to the problem and some related questions are given.

The modified Cauchy and some other problems for systems of ordinary differential equations with singularities, i.e. for problem (1.1),(1.3), are investigated, for example, in [7-10] (see also the references therein).

The singularity of system (1.1) is considered in the sense that the matrix-function $P$ or vector-function $q$ are generally not integrable at the point $t_{0}$, i.e. on some $] a, b[$ from $I$ such that $\left.t_{0} \in\right] a, b[$. So that, in general, the solution of problem (1.1), (1.3) is not continuous at the point $t_{0}$ and, therefore, it is not a solution in the classical sense. But its restriction on the every interval from $I_{t_{0}}$ is a solution of system (1.1) in the classical sense. To illustrate this we give the following example from [8].

Let $\alpha>0$ and $\varepsilon \in] 0, \alpha[$. Then the problem

$$
\frac{d x}{d t}=-\frac{\alpha x}{t}+\varepsilon|t|^{\varepsilon-1-\alpha}, \lim _{t \rightarrow 0}\left(t^{\alpha} x(t)\right)=0
$$

has the unique solution $x(t)=|t|^{\varepsilon-\alpha} \operatorname{sgn} t$. This function is not solution of the equation on the set $I=\mathbb{R}$, but its restrictions on $]-\infty, 0[$ and $] 0,+\infty[$ are solutions of the one.

As we know, such a problem for the impulsive differential system (1.1), (1.2) has not been investigated. So, the present investigation is quite topical.

The theory of the regular impulsive differential equations has been investigated in earlier papers (see, for example, $[1-6,11,12]$ and references therein). As to singular case, the corresponding theory, as we know, is far enough from deep research. Some boundary value problems for linear impulsive systems with singularities are investigated in [4]).

In the present paper, we give sufficient conditions for the unique solvability of problem (1.1) - (1.3). Analogous results for the analogous problem for systems of ordinary differential equations with singularities belong to I. Kiguradze (see [8-10]).

In the paper the use will be made of the following notation and definitions.

$\mathbb{N}=\{1,2, \ldots\}$.

$\mathbb{R}=]-\infty,+\infty\left[, \mathbb{R}_{+}=[0,+\infty[,[a, b]\right.$ and $] a, b[(a, b \in \mathbb{R})$ are, respectively, closed and open intervals.

$\mathbb{R}^{n \times m}$ is the space of all real $n \times m$ matrices $X=\left(x_{i, j}\right)_{i, j=1}^{n, m}$ with the norm

$$
\|X\|=\max _{j=1, \ldots, m} \sum_{i=1}^{n}\left|x_{i j}\right|
$$


$O_{n \times m}($ or $O$ ) is the zero $n \times m$ matrix.

If $X=\left(x_{i k}\right)_{i, k=1}^{n, m} \in \mathbb{R}^{n \times m}$, then

$$
\begin{gathered}
|X|=\left(\left|x_{i k}\right|\right)_{i, k=1}^{n, m}, \quad[X]_{+}=\frac{|X|+X}{2}, \quad[X]_{-}=\frac{|X|-X}{2} . \\
\mathbb{R}_{+}^{n \times m}=\left\{\left(x_{i j}\right)_{i, j=1}^{n, m}: \quad x_{i j} \geq 0(i=1, \ldots, n ; j=1, \ldots, m)\right\} .
\end{gathered}
$$

$\mathbb{R}^{n}=\mathbb{R}^{n \times 1}$ is the space of all real column $n$-vectors $x=\left(x_{i}\right)_{i=1}^{n}$.

If $X \in \mathbb{R}^{n \times n}$, then $X^{-1}, \operatorname{det} X$ and $r(X)$ are, respectively, the matrix inverse to $X$, the determinant of $X$ and the spectral radius of $X ; I_{n}$ is the identity $n \times n$-matrix.

The inequalities between the matrices are understood componentwise.

A matrix-function is said to be continuous, integrable, nondecreasing, etc., if each of its components is such.

$X(t-)$ and $X(t+)$ are, respectively, the left and the right limits of the matrixfunction $X:[a, b] \rightarrow \mathbb{R}^{n \times m}$ at the point $t$.

$A C([a, b] ; D)$, where $D \subset \mathbb{R}^{n \times m}$, is the set of all absolutely continuous matrixfunctions $X:[a, b] \rightarrow D$.

$A C_{\text {loc }}\left(I ; \mathbb{R}^{n \times m}\right)$ is the set of all matrix-functions $X: I \rightarrow \mathbb{R}^{n \times m}$ for which the restriction on $[a, b]$ belongs to $A C\left([a, b] ; \mathbb{R}^{n \times m}\right)$ for every closed interval $[a, b]$ from $I$;

$A C_{l o c}\left(I_{t_{0}, T} ; D\right)$, where $I_{t_{0}, T}=I_{t_{0}} \backslash T$, is the set of all matrix-functions $X: I_{t_{0}} \rightarrow D$ whose restrictions to an arbitrary closed interval $[a, b]$ from $I_{t_{0}, T}$ belong to $A C([a, b] ; D)$.

If $\alpha<\beta$ then $N_{\alpha, \beta}=\left\{l \in \mathbb{N}: \alpha \leq \tau_{l}<\beta\right\}$.

$L([a, b] ; D)$ is the set of all integrable matrix-functions $X:[a, b] \rightarrow D$.

$L_{l o c}\left(I_{t_{0}} ; D\right)$ is the set of all matrix-functions $X: I_{t_{0}} \rightarrow D$ whose restrictions to an arbitrary closed interval $[a, b]$ from $I_{t_{0}}$ belong to $L([a, b] ; D)$.

$E\left(M ; \mathbb{R}^{n \times m}\right)$, where $M \subset \mathbb{N}$, is the set of all discrete matrix-functions from $M$ into $\mathbb{R}^{n \times m}$

A vector-functions $x \in A C_{l o c}\left(I_{t_{0}, T} ; \mathbb{R}^{n}\right)$ is said to be a solution of system (1.1), (1.2) if $x^{\prime}(t)=P(t) x(t)+q(t)$ for a.a. $t \in I_{t_{0}, T}$ and there exist the onesided limits $x\left(\tau_{l}-\right)$ and $x\left(\tau_{l}+\right)(l=1,2, \ldots)$ such that equalities (1.2) hold.

Without loss of generality we can assume that the solution $x$ of the impulsive differential system (1.1), (1.2) is continuous from the left in the points of the impulses actions $\tau_{l}(l=1,2, \ldots)$, i.e. $x\left(\tau_{l}\right)=x\left(\tau_{l}-\right)(l=1,2, \ldots)$.

We assume that

$$
\operatorname{det}\left(I_{n}+G(l)\right) \neq 0 \quad(l=1,2, \ldots) .
$$

The above inequalities guarantee the unique solvability of the Cauchy problem for the corresponding nonsingular systems, i.e. for the case when $P \in L_{l o c}\left(I ; \mathbb{R}^{n \times n}\right)$ and $q \in L_{l o c}\left(I ; \mathbb{R}^{n}\right)($ see $[1,5,6,12])$.

Let $P_{0} \in L_{l o c}\left(I_{t_{0}} ; \mathbb{R}^{n \times n}\right)$ and $G_{0} \in E\left(\mathbb{N} ; \mathbb{R}^{n \times n}\right)$. Then a matrix-function $C_{0}: I_{t_{0}} \times$ $I_{t_{0}} \rightarrow \mathbb{R}^{n \times n}$ is said to be the Cauchy matrix of the homogeneous impulsive differential 
system

$$
\begin{gathered}
\frac{d x}{d t}=P_{0}(t) x, \\
x\left(\tau_{l}+\right)-x\left(\tau_{l}-\right)=G_{0}(l) x\left(\tau_{l}\right) \quad(l=1,2, \ldots)
\end{gathered}
$$

if for every interval $J \subset I_{t_{0}}$ and $\tau \in J$, the restriction of the matrix-function $C_{0}(., \tau)$ : $I_{t_{0}} \rightarrow \mathbb{R}^{n \times n}$ on $J$ is the fundamental matrix of system (1.4),(1.5) satisfying the condition $C_{0}(\tau, \tau)=I_{n}$. Therefore, $C_{0}$ is the matrix Cauchy of system (1.4), (1.5) if and only if the restriction of $C_{0}$ on $J \times J$, for every interval $J \subset I_{t_{0}}$, is the matrix Cauchy of the system in the sense of definition given in [8].

We assume

$$
I_{t_{0}}(\delta)=\left[t_{0}-\delta, t_{0}+\delta\right] \cap I_{t_{0}}
$$

for every $\delta>0$.

We consider problem (1.1) - (1.3) only in the case where $t_{0}=\sup I$ and $\tau_{l}<\tau_{l+1}$ $(l=1,2, \ldots)$. Similarly, we can investigate the case where $t_{0}=\inf I$ and $\tau_{l+1}<\tau_{l}$ $(l=1,2, \ldots)$. The general case $\left.t_{0} \in\right] \inf I, \sup I[$ will be reduced to the given two cases.

\section{FORMULATION OF THE MAIN RESUlTS}

Theorem 1. Let there exist a matrix-function $P_{0} \in L_{l o c}\left(I_{t_{0}, T} ; R^{n \times n}\right)$, discrete matrixfunction $G_{0} \in E\left(\mathbb{N} ; \mathbb{R}^{n \times n}\right)$ and constant matrices $B_{0}, B \in \mathbb{R}_{+}^{n \times n}$ such that the conditions

$$
\begin{aligned}
\operatorname{det}\left(I_{n}+G_{0}(l)\right) & \neq 0(l=1,2, \ldots), \\
r(B) & <1
\end{aligned}
$$

and the estimates

$$
\left|C_{0}(t, \tau)\right| \leq H(t) B_{0} H^{-1}(\tau) \text { for } t \leq \tau, t, \tau \in\left[t_{0}-\delta, t_{0}[\right.
$$

and

$$
\begin{aligned}
& \left|\int_{t}^{t_{0}}\right| C_{0}(t, \tau)\left(P(s)-P_{0}(s)\right) H(s)|d s| \\
& +\sum_{l \in N_{t, t_{0}}}\left|C_{0}\left(t, \tau_{l}\right)\left(I_{n}+G_{0}(l)\right)^{-1}\left(G(l)-G_{0}(l)\right) H\left(\tau_{l}\right)\right| \leq H(t) B \\
& \qquad \text { for } t \in\left[t_{0}-\delta, t_{0}[\right.
\end{aligned}
$$

hold for some $\delta>0$, where $C_{0}$ is the Cauchy matrix of system (1.4), (1.5). Let, moreover, 


$$
\begin{aligned}
\lim _{t \rightarrow t_{0}} \| \int_{t}^{t_{0}} H^{-1}(s) C_{0}(t, s) q(s) d s & \\
& \quad+\sum_{l \in N_{t, t_{0}}} H^{-1}\left(\tau_{l}\right) C_{0}\left(t, \tau_{l}\right)\left(I_{n}+G_{0}(l)\right)^{-1} g(l) \|=0 .
\end{aligned}
$$

Then problem (1.1) - (1.3) has a unique solution.

Theorem 2. Let there exist a constant matrix $B=\left(b_{i k}\right)_{i, k=1}^{n} \in \mathbb{R}_{+}^{n \times n}$ such that condition (2.2) hold, and the estimates

$$
\begin{aligned}
& c_{i}(t, \tau) \leq b_{0} \frac{h_{i}(t)}{h_{i}(\tau)} \text { for } t \leq \tau, t, \tau \in\left[t_{0}-\delta, t_{0}[(i=1, \ldots, n),\right. \\
& \left|\int_{t}^{t_{0}} c_{i}(t, \tau) h_{i}(\tau)\left[p_{i i}(\tau)\right]_{-} d \tau+\sum_{l \in N_{t, t_{0}}} c_{i}\left(t_{l}, \tau_{l}\right) h_{i}\left(\tau_{l}\right)\left[g_{i i}(l)\right]_{-}\right| \\
& \quad \leq b_{i i} h_{i}(t) \text { for } t \in\left[t_{0}-\delta, t_{0}[(i=1, \ldots, n),\right. \\
& \quad\left|\int_{t}^{t_{0}} c_{i}(t, \tau) h_{k}(\tau)\right| p_{i k}(\tau) \mid d \tau \\
& \quad+\sum_{l \in N_{t, t_{0}}} c_{i}\left(t, \tau_{l}\right)\left[g_{i i}(l)\right]_{+} \cdot\left(1+\left[g_{i i}(l)\right]_{+}\right)^{-1} h_{k}\left(\tau_{l}\right) g_{i k}(l) \mid \\
& \quad \leq b_{i k} h_{i}(t) \text { for } t \in\left[t_{0}-\delta, t_{0}[(i \neq k ; i, k=1, \ldots, n)\right.
\end{aligned}
$$

hold for some $b_{0}>0$ and $\delta>0$. Let, moreover,

$$
\begin{aligned}
& \lim _{t \rightarrow t_{0}}\left(\int_{t}^{t_{0}} \frac{c_{i}(t, \tau)}{h(t)} q(\tau) d \tau\right. \\
& \left.+\sum_{l \in N_{t, t_{0}}} \frac{c_{i}\left(t, \tau_{l}\right)}{h_{i}(t)}\left[g_{i i}(l)\right]_{+} \cdot\left(1+\left[g_{i i}(l)\right]_{+}\right)^{-1} g(l)\right)=0 \quad(i=1, \ldots, n),
\end{aligned}
$$

where $c_{i}$ is the Cauchy function of the impulsive differential equations

$$
\begin{gathered}
\frac{d x}{d t}=p_{0 i}(t) x, \\
x\left(\tau_{l}+\right)-x\left(\tau_{l}-\right)=g_{0 i}(l) x\left(\tau_{l}\right)(l=1,2, \ldots),
\end{gathered}
$$

here

$$
p_{0 i}(t) \equiv\left[p_{i i}(t)\right]_{+}, \quad g_{0 i}(l) \equiv\left[g_{i i}(l)\right]_{+}(i=1, \ldots, n) .
$$

Then problem (1.1) - (1.3) has a unique solution. 
Remark 1. The Cauchy functions $c_{i}(t, \tau)(i=1, \ldots, n)$ have the form

$$
c_{i}(t, \tau)= \begin{cases}\exp \left(\int_{\tau}^{t}\left[p_{i i}(s)\right]_{+} d s\right) \prod_{l \in T_{\tau, t}}\left(1+\left[g_{i i}(l)\right]_{+}\right) & \text {if } t>\tau, \\ \exp \left(\int_{\tau}^{t}\left[p_{i i}(s)\right]_{+} d s\right) \prod_{l \in T_{t, \tau}}\left(1+\left[g_{i i}(l)\right]_{+}\right)^{-1} & \text { if } t<\tau, \\ 1 & \text { if } t=\tau\end{cases}
$$

for $t, \tau \in I$.

Remark 2. In Theorems 1 and 2, the strong inequality (2.2) can not replace by a non-strong one. We give the corresponding example from [8] for the ordinary differential case, i.e., when $G(l) \equiv O_{n \times n}$ and $g(l) \equiv 0_{n}$.

On the interval $]-1,0[$ consider the problem

$$
\begin{gathered}
\frac{d x}{d t}=\frac{x}{t}+\frac{1}{|\ln | t||}, \\
\lim _{t \rightarrow 0} \frac{x(t)}{t}=0 .
\end{gathered}
$$

Every solution of equation (2.11) has the form

$$
x(t)=c t-t \ln |\ln | t|| \quad(c \in \mathbb{R}) .
$$

So that, problem (2.11), (2.12) is not solvable. On the other hand, the Cauchy function $c(t, \tau)$ has the form $c(t, \tau)=t \tau^{-1}$ for $t \leq \tau<0$ and the conditions of Theorem 1, except of condition (2.2), are fulfilled (on ] $-1,0\left[\right.$ ) for $n=1, P(t) \equiv t^{-1}$, $q(t) \equiv|\ln | t||^{-1}$ and $h(t) \equiv t$ only for the case where $B \geq 1$, i.e., when $r(B) \geq 1$.

\section{AUXILIARY PROPOSITIONS}

We use the lemma on the a priory estimate of the solutions of system (1.1), (1.2) (see below, Lemma 2). To prove the lemma, we use a Cauchy formula to represent solutions of the impulsive differential systems.

Lemma 1 (Variation-of-constants formula). Let $G_{*} \in E\left(\mathbb{N} ; \mathbb{R}^{n \times n}\right)$ be such that

$$
\operatorname{det}\left(I_{n}+G_{*}(l)\right) \neq 0(l=1,2, \ldots) .
$$

Then every solution of the system

$$
\begin{gathered}
\frac{d x}{d t}=P_{*}(t) x+q_{*}(t) \text { for a. } a . \quad t \in I, \\
x\left(\tau_{l}+\right)-x\left(\tau_{l}-\right)=G_{*}(l) x\left(\tau_{l}\right)+g_{*}(l) \quad(l=1,2, \ldots),
\end{gathered}
$$


where $P_{*} \in L_{l o c}\left(I ; \mathbb{R}^{n s \times n}\right), q_{*} \in L_{l o c}\left(I ; \mathbb{R}^{n}\right)$ and $g_{*} \in E\left(\mathbb{N} ; \mathbb{R}^{n \times n}\right)$, admits the representation

$$
\begin{gathered}
x(t)=C_{*}(t, \tau) x(\tau)+\int_{\tau}^{t} C_{*}(t, s) q_{*}(s) d s \\
-\sum_{l \in N_{\tau, t}} C_{*}\left(t, \tau_{l}\right) G_{*}(l)\left(I_{n}+G_{*}(l)\right)^{-1} g_{*}(l) \text { for } \tau<t, \tau, t \in I,
\end{gathered}
$$

where $C_{*}$ is the Cauchy matrix of the homogeneous system

$$
\begin{gathered}
\frac{d x}{d t}=P_{*}(t) x \text { for } a . a . \quad t \in I, \\
x\left(\tau_{l}+\right)-x\left(\tau_{l}-\right)=G_{*}(l) x\left(\tau_{l}\right)(l=1,2, \ldots) .
\end{gathered}
$$

Representation (3.1) is proved for example in [5].

Lemma 2. Let the matrix-functions $P_{0} \in L_{l o c}\left(I_{t_{0}} ; \mathbb{R}^{n \times n}\right), G_{0} \in E\left(\mathbb{N} ; \mathbb{R}^{n \times n}\right)$ and the constant matrices $B_{0}$ and $B$ from $\mathbb{R}_{+}^{n \times n}$ be such that conditions (2.1)-(2.4) hold for some $\delta>0$, where $C_{0}$ is the Cauchy matrix of system (1.4), (1.5). Let, moreover,

$$
\begin{gathered}
\gamma(t)=\sup \left\{\| \int_{\tau}^{t_{0}} \mid H^{-1}(s) C_{0}(t, s) q(s) d s\right. \\
\left.+\sum_{l \in N_{t, t_{0}}} H^{-1}\left(\tau_{l}\right) C_{0}\left(t, \tau_{l}\right) G_{0}(l)\left(I_{n}+G_{0}(l)\right)^{-1} g(l) \|: t \leq \tau<t_{0}\right\}<+\infty \\
\text { for } t \in\left[t_{0}-\delta, t_{0}[.\right.
\end{gathered}
$$

Then every solution $x$ of system (1.1), (1.2) admits the estimate

$$
\begin{gathered}
\left\|H^{-1}(t) x(t)\right\| \leq \rho\left(\left\|B_{0}\right\|\left\|H^{-1}\left(\tau_{0}\right) x\left(\tau_{0}\right)\right\|+\gamma(t)\right) \\
\text { for } t \in J, \quad t<\tau_{0},
\end{gathered}
$$

where $\rho=\left\|\left(I_{n}-B\right)^{-1}\right\|$, and $J \subset\left[t_{0}-\delta, t_{0}\left[\right.\right.$ and $\tau_{0} \in J$ are arbitrary interval and point, respectively.

Proof. Let $x=\left(x_{i}\right)_{i=1}^{n}$ be an arbitrary solution of system (1.1), (1.2) on the $J$. Then $x$ satisfies the impulsive system

$$
\begin{gathered}
\frac{d x}{d t}=P_{0}(t) x+\left(P(t)-P_{0}(t)\right) x+q(t) \text { for a. a. } t \in I_{t_{0}, T}, \\
x\left(\tau_{l}+\right)-x\left(\tau_{l}-\right)=G_{0}(l) x\left(\tau_{l}\right)+\left(G(l)-G_{0}(l)\right) x\left(\tau_{l}\right)+g(l)(l=1,2, \ldots) .
\end{gathered}
$$

Let the vector-function $z(t)=\left(z_{i}(t)\right)_{i=1}^{n}$ is defined by

$$
z(t) \equiv H^{-1}(t) x(t) \text {. }
$$


According to variations-of-constants formula (3.1) we have

$$
\begin{gathered}
x(t)=C_{0}\left(t, \tau_{0}\right) x\left(\tau_{0}\right)+\int_{\tau_{0}}^{t} C_{0}(t, s) q_{*}(s) d s \\
-\sum_{l \in N_{t,} \tau_{0}} C_{0}\left(t, \tau_{l}\right) G_{0}(l)\left(I_{n}+G_{0}(l)\right)^{-1} g_{*}(l) \text { for } t \in J, t<\tau_{0},
\end{gathered}
$$

where

$$
q_{*}(t) \equiv\left(P(t)-P_{0}(t)\right) x(t)+q(t) \text { and } g_{*}(l) \equiv\left(G(l)-G_{0}(l)\right) x\left(\tau_{l}\right)+g(l) .
$$

Therefore,

$$
\begin{gathered}
z(t)=H^{-1}(t) C_{0}\left(t, \tau_{0}\right) x\left(\tau_{0}\right)+H^{-1}(t) \int_{\tau_{0}}^{t} C_{0}(t, s) q_{*}(s) d s \\
-H^{-1}(t) \sum_{l \in N_{t, \tau_{0}}} C_{0}\left(t, \tau_{l}\right) G_{0}(l)\left(I_{n}+G_{0}(l)\right)^{-1} g_{*}(l) \text { for } t \in J, t<\tau_{0} .
\end{gathered}
$$

Let the components of the vector-function $y(t)=\left(y_{i}(t)\right)_{i=1}^{n}$ be defined by

$$
y_{i}(t)=\sup \left\{\left|z_{i}(s)\right|: t \leq s \leq \tau_{0}\right\} \text { for } t \in J(i=1, \ldots, n) .
$$

In view of (3.4), take into account (2.3) and (2.4), it is not difficult to verify that

$$
\begin{aligned}
& |z(t)| \leq H^{-1}(t)\left|C_{0}\left(t, \tau_{0}\right) x\left(\tau_{0}\right)\right| \\
& +H^{-1}(t) \int_{t}^{\tau_{0}}\left|C_{0}(t, s)\left(P(s)-P_{0}(s)\right) H(s)\right||z(s)| d s \\
& +H^{-1}(t) \sum_{l \in N_{t, \tau_{0}}}\left|C_{0}\left(t, \tau_{l}\right) G_{0}(l)\left(I_{n}+G_{0}(l)\right)^{-1}\left(G(l)-G_{0}(l)\right) H\left(\tau_{l}\right)\right|\left|z\left(\tau_{l}\right)\right| \\
& +H^{-1}(t) f(t) \leq B_{0}\left|H^{-1}\left(\tau_{0}\right) x\left(\tau_{0}\right)\right|+B y(t)+H^{-1}(t) f(t) \\
& \text { for } t \in J, t<\tau_{0} .
\end{aligned}
$$

where

$$
f(t) \equiv \int_{t}^{\tau_{0}}\left|C_{0}(t, s) q(s)\right| d s+\sum_{l \in N_{t, \tau_{0}}}\left|C_{0}\left(t, \tau_{l}\right) G_{0}(l)\left(I_{n}+G_{0}(l)\right)^{-1} g(l)\right| .
$$

Therefore, thanks to (3.2) we find

$$
y(t) \leq B_{0}\left|H^{-1}\left(\tau_{0}\right) x\left(\tau_{0}\right)\right|+B y(t)+\bar{\gamma}(t) \text { for } t \in J, t<\tau_{0}
$$

and

$$
\left(I_{n}-B\right) y(t) \leq B_{0}\left|H^{-1}\left(s_{0}\right) x\left(s_{0}\right)\right|+\bar{\gamma}(t) \text { for } t \in J, \quad t<\tau_{0},
$$

where $\bar{\gamma}(t)$ is a vector-function with components $\gamma_{i}(t) \equiv \gamma(t)(i=1, \ldots, n)$. 
From this, in view of (2.2) and nonnegativity of matrix $B$, we have

$$
y(t) \leq\left(I_{n}-B\right)^{-1}\left(B_{0}\left|H^{-1}\left(s_{0}\right) x\left(s_{0}\right)\right|+g(t)\right) \text { for } t \in J, \quad t<\tau_{0} .
$$

Hence estimate (3.3) holds.

\section{PROOFS OF RESUlTS}

Proof of Theorem 1. In the first place, we note that by (2.5) estimate (3.2) holds and

$$
\lim _{t \rightarrow t_{0}} \gamma(t)=0
$$

where the function $\gamma(t)$ is defined as in Lemma 2.

Let $t_{k} \in\left[t_{0}-\delta, t_{0}[(k=1,2, \ldots)\right.$ be some increasing sequence such that

$$
\lim _{k \rightarrow+\infty} t_{k}=t_{0}
$$

According to Theorem 2.1.1 from [5], for every natural $k$, system (1.1), (1.2) has a unique solution $x_{k}$ defined on the interval $\left[t_{0}-\delta, t_{0}[\right.$ and satisfying the condition

$$
x_{k}\left(t_{k}\right)=0 .
$$

Moreover, due to Lemma 2 we have the estimates

$$
\left\|H^{-1}(t) x_{k}(t)\right\| \leq \rho \gamma(t) \text { for } t_{1} \leq t \leq t_{k}(k=1,2, \ldots),
$$

where $\rho=\left\|\left(I_{n}-B\right)^{-1}\right\| \gamma\left(t_{1}\right)$. In particular, from (4.3) it follows that

$$
\left\|x_{k}\left(t_{1}\right)\right\| \leq \rho_{0} \quad(k=1,2, \ldots),
$$

where $\rho_{0}=\rho\left\|H\left(t_{1}\right)\right\| \gamma\left(t_{1}\right)$. So with out of generality we can assume that the sequence $x_{k}\left(t_{1}\right)(k=1,2, \ldots)$ converges. Let

$$
\lim _{k \rightarrow+\infty} x_{k}\left(t_{1}\right)=c_{0} .
$$

By the theorems on the well-posedness of the Cauchy problem (see [5]) we conclude

$$
\lim _{k \rightarrow+\infty} x_{k}(t)=x(t)
$$

uniformly on the every closed interval from $\left[t_{0}-\delta, t_{0}[\right.$, where $x$ is a solution of system (1.1), (1.2) under the condition

$$
x\left(t_{1}\right)=c_{0} .
$$

On the other hand, thanks to (4.2) and (4.3) we find that

$$
\left\|H^{-1}(t) x(t)\right\| \leq \rho \gamma(t) \text { for } t_{1} \leq t<t_{0} .
$$

From this, by (4.1) we conclude that the vector-function $x$ is a solution of problem $(1.1)-(1.3)$ on the interval $\left[t_{0}-\delta, t_{0}[\right.$. 
Now, let us show that problem (1.1) - (1.3) has a unique solution on the interval $\left[t_{0}-\delta, t_{0}\left[\right.\right.$. Let, $x_{*}$ be an arbitrary solution of the problem. Then the vector-function $x_{0}(t)=x(t)-x_{*}(t)$ will be a solution of the homogeneous system

$$
\begin{gathered}
\frac{d x}{d t}=P(t) x \text { for a. a. } t \in I_{t_{0}, T}, \\
x\left(\tau_{l}+\right)-x\left(\tau_{l}-\right)=G(l) x\left(\tau_{l}\right) \quad(l=1,2, \ldots)
\end{gathered}
$$

under the condition

$$
\lim _{t \rightarrow t_{0}}\left(H^{-1}(t) x_{0}(t)\right)=0 .
$$

In view of estimate (3.3) of Lemma 2 , for every $\tau_{0} \in\left[t_{0}-\delta, t_{0}[\right.$, we find

$$
\left\|x_{0}\left(t_{0}+\delta\right)\right\| \leq \rho_{0}\left\|H\left(t_{0}+\delta\right)\right\| \cdot\left\|H^{-1}\left(\tau_{0}\right) x_{0}\left(\tau_{0}\right)\right\| \text { for } t_{0}+\delta \leq t \leq \tau_{0},
$$

where $\rho_{0}=\left\|\left(I_{n}-B\right)^{-1}\right\| \cdot\left\|B_{0}\right\|$. Passing to the limit as $\tau_{0} \rightarrow t_{0}$ in the last estimate and taking into account (4.4) we get

$$
x\left(t_{0}+\delta\right)=0 .
$$

Since the matrix-function $P$ is integrable at the point $t_{0}+\delta$, i.e., we have regular case, by the above-mentioned theorem from [5] problem $\left(1.1_{0}\right),\left(1.2_{0}\right) ;(4.5)$ has only the trivial solution and, therefore, $x(t) \equiv x_{*}(t)$.

Proof of Theorem 2. Let us assume

$$
P_{0}(t) \equiv \operatorname{diag}\left(\left[p_{11}(t)\right]_{+}, \ldots,\left[p_{n n}(t)\right]_{+}\right)
$$

and

$$
G_{0}(t) \equiv \operatorname{diag}\left(\left[g_{11}(l)\right]_{+}, \ldots,\left[g_{n n}(l)\right]_{+}\right) .
$$

Then the Cauchy matrix of system (1.4), (1.5) has the form

$$
C(t, \tau) \equiv \operatorname{diag}\left(c_{1}(t, \tau), \ldots, c_{n}(t, \tau)\right),
$$

where the functions $c_{i}(t, \tau)(i=1, \ldots, n)$ are defined by (2.10).

In addition, note that due to $(2.10)$ we have

$$
c_{i}(t, \tau)>0 \text { for } t \leq \tau, t, \tau \in\left[t_{0}-\delta .\right.
$$

hold. By this, conditions (2.6) - (2.9) we conclude that conditions (2.3), (2.4) and (2.5) of Theorem 1 are valid. Hence the theorem immediately follows from Theorem 1. 


\section{REFERENCES}

[1] A. M. Samoilenko and N. A. Perestyuk, Impulsive differential equations. River Edge, NJ: World Scientific Series on Nonlinear Science. Series A: Monographs and Treatises, 14. World Scientific Publishing Co., Inc.,, 1995. doi: https://doi.org/10.1142/2892.

[2] S. Akhalaia, M. Ashordia, and N. Kekelia, "On the necessary and sufficient conditions for the stability of linear generalized ordinary differential, linear impulsive and linear difference systems." Georgian Math. J., vol. 16, no. 4, pp. 597-616, 2009, doi: https://doi.org/10.1515/GMJ.2009.597.

[3] M. Ashordia, "On the general and multipoint boundary value problems for linear systems of generalized ordinary differential equations, linear impulse and linear difference systems." Mem. Differential Equations Math. Phys., vol. 36, pp. 1-80, 2005.

[4] M. Ashordia, "On the Two-Point Boundary Value Problems for Linear Impulsive Systems with Singularities." Georgian Math. J., vol. 19, no. 1, pp. 19-40, 2012, doi: https://doi.org/10.1515/gmj-2011-0053.

[5] M. Ashordia, "The Initial Problem for Linear Systems of Generalized Ordinary Differential Equations, Linear Impulsive and Ordinary Differential Systems. Numerical Solvability." Mem. Differential Equations Math. Phys., vol. 78, pp. 1-162, 2019.

[6] D. D. Bainov and P. S. Simeonov, Impulsive differential equations: asymptotic properties of the solutions. Singapore: Series on Advances in Mathematics for Applied Sciences. 28. World Scientific, 1995. doi: https://doi.org/10.1142/2413.

[7] V. A. Chechik, "Untersuchung von Systemen gewöhnlicher Differentialgleichungen mit einer Singularität (in Russian)." Tr. Mosk. Mat. Obshch., vol. 8, pp. 155-198, 1959.

[8] I. Kiguradze, The initial value problem and boundary value problems for systems of ordinary differential equations. Vol. I. Linear theory. Tbilisi: Metsniereba, 1997.

[9] I. T. Kiguradze, "On the Cauchy problem for singular systems of ordinary differential equations (in Russian)." Differential'nye Uravnenija, vol. 1, pp. 1271-1291, 1965.

[10] I. T. Kiguradze, "On a singular Cauchy problem for systems of linear ordinary differential equations (in Russian)." Differ. Uravn., vol. 32, no. 2, pp. 171-179, 1996.

[11] N. A. Perestyuk and V. A. Plotnikov and A. M. Samoilenko and N. V. Skripnik, Differential equations with impulse effects. Multivalued right-hand sides with discontinuities. Berlin: De Gruyter Studies in Mathematics 40. Walter de Gruyter \& Co., 2011.

[12] V. Lakshmikantham, Trends in the theory of impulsive differential equations. Athens, OH: Differential equations and applications, Vol. I, II (Columbus, OH, 1988), Ohio Univ. Press, 1989.

Authors' addresses

Malkhaz Ashordia

A. Razmadze Mathematical Institute, Some Department, 6. Tamarashvili Str., 0177 Tbilisi, Georgia, Sukhumi State University, 61, A. Politkovskaia Str., 0186 Tbilisi, Georgoa

E-mail address: ashordermi.ge, malkhaz.ashordiadtsu.ge

Nato Kharshiladze

Sukhumi State University, 61, A. Politkovskaia Str., 0186 Tbilisi, Georgoa

E-mail address: natokharshiladze@ymail.com 